\title{
On a Boundary Extrapolation Theorem by Kreiss
}

\author{
By Moshe Goldberg*
}

\begin{abstract}
A hardly known and very important result of Kreiss is proven explicitly: Outflow boundary extrapolation, which complements stable dissipative schemes for linear hyperbolic initial value problems, maintains stability. In view of this result, the Lax-Wendroff and the Gottlieb-Turkel schemes are applied to a test problem. As expected from the rate-of-convergence theory by Gustafsson, global order of accuracy is preserved if outflow boundary computations employ extrapolation of (local) accuracy of the same order.
\end{abstract}

1. Introduction. The initial value problem

$$
u_{t}=a u_{x} ; \quad a>0 ; \quad x \geqslant 0, t \geqslant 0 ; \quad u(x, 0)=f(x),
$$

is well posed in $L^{2}(0, \infty)$, and requires no boundary conditions at $x=0$. Yet, it is impossible to approximate the solution of $(1.1)$ by a difference scheme, which is not right-sided, without specifying boundary values at some points in a left neighborhood of $x=0$.

In this paper we consider general two-sided dissipative schemes which are stable for the pure Cauchy problem for $-\infty<x<\infty$. Our main purpose is to provide a proof for the following important result which was stated by Kreiss in 1965 [4, Theorem 5], but no detailed proof has been published. We show that if the required boundary values are defined by extrapolation of arbitrary order of accuracy, the numerical algorithm remains stable.

Of course, one may have instead of (1.1), the equation $u_{t}=a u_{x}$ with $a<0$, which defines a well-posed initial value problem in the quarter plane $\{x \leqslant 0, t \geqslant 0\}$, rather than in $\{x \geqslant 0, t \geqslant 0\}$. However, by employing the transformation $x \rightarrow-x$ it is clear that this problem goes over to the previous one. Consequently, we would find that the process of extrapolating to grid points at some right neighborhood of $x=0$, is stable.

To summarize, our aim is to show that by using a stable two-sided dissipative scheme together with an outflow extrapolation, to approximate a well-posed initial value problem in the proper quarter plane, overall stability is maintained. Again, since the cases $a>0$ and $a<0$ are analogous, it suffices to prove stability for difference approximations of (1.1), and the proof is given in Section 2.

The tool by which we carry out the analysis is Kreiss' stability theory for dissipative finite-difference approximations of mixed initial boundary value problems.

Received September 13, 1976.

AMS (MOS) subject classifications (1970). Primary 65M10; Secondary 65N10.

*This research was sponsored in part by the Air Force Office of Scientific Research, Air Force System Command, USAF, under Grant No. AFOSR-76-3046. 
This theory is given in [5], and we assume that the reader is familiar with this work.

In Section 3 we present numerical evidence to support the theoretical results.

We use two dissipative approximations: The well-known centered 3-point Lax-Wendroff scheme, [7], and a centered 5-point scheme by Gottlieb and Turkel, [2]. In particular, our computations verify that by using extrapolation of local order of accuracy which equals the global order of accuracy of the difference scheme, the global accuracy is preserved. The important question of convergence rate for mixed initial boundary value problems is discussed by Gustafsson, [3] .

The computations reported in this work were done on the IBM 360 machine, at the Campus Computing Network of the University of California, Los Angeles.

2. Stability Analysis. In order to solve the initial value problem (1.1) by a finitedifference scheme let us introduce a mesh-size $\Delta x>0, \Delta t>0$, such that $\lambda=\Delta t / \Delta x$ $=$ constant, and use the standard notation $x_{\nu}=v \Delta x, v_{\nu}(t)=v\left(x_{\nu}, t\right)$. Now consider a dissipative consistent approximation to (1.1) of the form

$$
v_{\nu}(t+\Delta t)=Q v_{\nu}(t), \quad v=1,2, \ldots,
$$

where

$$
Q=\sum_{j=-r}^{p} a_{j} E^{j}, \quad E v_{\nu}=v_{\nu+1},
$$

and initial values are given by

$$
v_{\nu}(0)=f_{\nu}, \quad \nu=1,2, \ldots
$$

Here the fixed coefficients $a_{j}$ depend on $a$ and $\lambda$, such that $a_{-r}, a_{p}$ do not vanish.

The assumption of dissipativity (in the sense of Kreiss) means that there exist a constant $\delta>0$ and a positive integer $\omega$, so that the amplification factor

$$
\hat{Q}(\xi)=\sum_{j=-r}^{p} a_{j} e^{i j \xi}, \quad-\pi \leqslant \xi \leqslant \pi,
$$

of the difference scheme, satisfies

$$
|\hat{Q}(\xi)| \leqslant 1-\delta|\xi|^{2 \omega}, \quad \forall|\xi| \leqslant \pi .
$$

Condition (2.5) guarantees, of course, the (strong) stability of the approximation, should it be applied to the pure initial value problem for $-\infty<x<\infty$.

Our final assumption, as indicated in the introduction, is that the scheme is two-sided, i.e., $r>0, p>0$. In fact, having dissipativity, our scheme must be twosided if we simply require that the difference operator $Q$ will be consistent with $u_{t}=a u_{x}$ for an arbitrary value of $a$-positive or negative. This result is given in Corollary 1 of [1].

Since $r>0$, it is evident that in order to apply the numerical approximation to (1.1), we have to specify, at each time step, boundary values $v_{\mu}(t), \mu=0,-1, \ldots$, $-r+1$. We do this by means of extrapolation, utilizing the Lagrange interpolation polynomial of degree $s-1, s \geqslant 1$, which has accuracy of order $s$.

In order to comply with Kreiss' formulation in [5], we should use the procedure 


$$
v_{\mu}(t)=\sum_{j=1}^{s} c_{j \mu} v_{j}(t), \quad \mu=0, \ldots,-r+1,
$$

where the Lagrange coefficients, $c_{j \mu}$, depend on $\mu$ and are given by

$$
\begin{gathered}
c_{j \mu}=\frac{W\left(x_{\mu}\right)}{\left(x_{\mu}-x_{j}\right) W^{\prime}\left(x_{j}\right)} ; \quad \mu=0, \ldots,-r+1, j=1, \ldots, s, \\
W(x)=\left(x-x_{1}\right) \cdots\left(x-x_{s}\right) .
\end{gathered}
$$

The fact that the $c_{j \mu}$ vary with $\mu$ causes some numerical and theoretical inconvenience which we eliminate as follows. Since there exists a unique polynomial of degree $s-1$ which coincides with a given function at $s$ given points, (2.6) is equivalent to extrapolating from $v_{1}(t), \ldots, v_{s}(t)$ to $v_{0}(t)$, and then from $v_{0}(t), \ldots, v_{s-1}(t)$ to $v_{-1}(t)$, etc. That is, (2.6) is equivalent to the fixed coefficient extrapolation algorithm

$$
v_{\mu}(t)=\sum_{j=1}^{s} c_{j} v_{\mu+j}(t), \quad \mu=0, \ldots,-r+1,
$$

where

$$
c_{j}=\frac{w_{\mu}\left(x_{\mu}\right)}{\left(x_{\mu}-x_{\mu+j}\right) W_{\mu}^{\prime}\left(x_{\mu+j}\right)}, \quad j=1, \ldots, s
$$

and

$$
W_{\mu}(x)=\left(x-x_{\mu+1}\right)\left(x-x_{\mu+2}\right) \cdots\left(x-x_{\mu+s}\right), \quad \mu=0, \ldots,-r+1 .
$$

It is straightforward to verify that regardless of $\mu$

$$
c_{j}=\left(\begin{array}{l}
s \\
j
\end{array}\right)(-1)^{j+1}, \quad j=1, \ldots, s .
$$

So our boundary conditions are

$$
v_{\mu}(t)=\sum_{j=1}^{s}\left(\begin{array}{l}
s \\
j
\end{array}\right)(-1)^{j+1} v_{\mu+j}(t), \quad \mu=0, \ldots,-r+1,
$$

and we finally write them in the convenient form

$$
\sum_{j=0}^{s}\left(\begin{array}{l}
s \\
j
\end{array}\right)(-1)^{j} v_{\mu+j}=0, \quad \mu=0, \ldots,-r+1 .
$$

Now the approximation to (1.1) is well defined, where the local error at the boundary extrapolation is $O\left(\Delta x^{s}\right)$.

Next denote by $H$ the space of all grid functions $w_{\nu}$, defined for $\nu>-r$, which fulfill the boundary condition (2.12), and which satisfy

$$
\sum_{v=-r+1}^{\infty}\left|w_{\nu}\right|^{2} \Delta x<\infty .
$$

Upon defining inner product and norm by

$$
(u, v)=\sum_{v=-r+1}^{\infty} \bar{u}_{\nu} v_{\nu} \Delta x, \quad\|w\|^{2}=(w, w),
$$

$H$ becomes a Hilbert space. 
According to these definitions, we may present our difference approximations in the form

$$
v(t+\Delta t)=G v(t), \quad v(t), v(t+\Delta t) \in H
$$

where $G$ is a linear bounded operator in $H$ defined by (2.1) together with (2.12).

We say that the algorithm in (2.15) is stable if there exists a constant $K$, such that

$$
\|v(t)\| \leqslant K\|v(0)\|, \quad \forall t=m \Delta t \text { and } v(0) \in H .
$$

The above description follows Kreiss' representation in [5], so all the results of [5] hold, and we first rephrase the Main Theorem of [5] as follows: The finite-difference approximation is stable if the operator $G$ has no eigenvalues $z$ with $|z| \geqslant 1$, $z \neq 1$, and if $z=1$ is not a generalized eigenvalue of $G$.

The concept of a generalized eigenvalue is discussed in Section 1 of [5], and in the remainder of this section we shall show that for our problem, the Main Theorem is satisfied.

In order to check whether a given $z$ with $|z| \geqslant 1$ is an eigenvalue of $G$, we consider the characteristic equation of the difference operator $Q$,

$$
\operatorname{det}\left[\sum_{j=-r}^{p} a_{j} k^{j}-z\right]=0 .
$$

By Lemma 2 of [5], Eq. (2.17) with $z \neq 1,|z| \geqslant 1$, has $r+p$ roots $\kappa$; $r$ of them with $0<|\kappa|<1$ and $p$ with $|\kappa|>1$. Moreover, according to the proof of Lemma 7 of [5], as $z \rightarrow 1(|z| \geqslant 1, z \neq 1)$, precisely one root $\kappa$ tends to 1 ; and this root approaches 1 from inside the unit disc if and only if $a<0$. In our case, the coefficient $a$ in (1.1) is positive, so no root $k$ of (2.17) tends to 1 from inside the unit disc. Hence, $z=1$ is not a generalized eigenvalue of $G$, and it remains to verify that $z$ with $|z| \geqslant 1, z \neq 1$, is not an ordinary eigenvalue of $G$.

Suppose $z_{0}$ with $\left|z_{0}\right| \geqslant 1, z_{0} \neq 1$, is an eigenvalue of $G$, with a corresponding eigenvector $g \in H$. That is, $G g=z_{0} g$, or more specifically, due to the definition of $G, g$ must satisfy the relations

$$
\left(Q-z_{0}\right) g_{\nu}=0, \quad v=1,2, \ldots,
$$

and the boundary conditions

$$
\sum_{j=0}^{s}\left(\begin{array}{l}
s \\
j
\end{array}\right)(-1)^{j} g_{\mu+j}=0, \quad \mu=0,-1, \ldots,-r+1 .
$$

Take the characteristic equation with $z=z_{0}$, and let $\kappa_{i}, i=1, \ldots, q$, be all its distinct roots which satisfy $\left|\kappa_{i}\right|<1$, each with multiplicity $\boldsymbol{\gamma}_{i}$. We know that there are $r$ such roots, so

$$
\sum_{i=1}^{q} \gamma_{i}=r
$$

The most general solution of the ordinary difference equation (2.18), which belongs to $H$, is known to be 


$$
g_{\nu}=\sum_{i=1}^{q} \sum_{k=1}^{\gamma_{i}} \sigma_{i, k} \nu^{k-1} \kappa_{i}^{\nu}, \quad \nu \geqslant-r+1,
$$

where the $r$ coefficients $\sigma_{i, k}$ are arbitrary. We still have to verify that the solution in (2.21) satisfies the boundary conditions (2.19). So, we insert (2.21) into (2.19) and after a simple rearrangement we get

$$
\sum_{i=1}^{q} \sum_{k=1}^{\gamma_{i}}\left[\sum_{j=0}^{s}\left(\begin{array}{l}
s \\
j
\end{array}\right)(-1)^{j}(\mu+j)^{k-1} \kappa_{i}^{\mu+j}\right] \sigma_{i, k}=0, \quad \mu=0, \ldots,-r+1
$$

In (2.22) we have a homogeneous linear system of $r$ equations for the $r$ unknowns $\sigma_{i, k}$. Denoting the coefficient matrix by $E$, it can be shown, by elementary column operations, that $E$ reduces to a generalized Vandermonde. Hence, $\operatorname{det} E$ is proportional to an expression of the form

$$
\left[\prod_{i=1}^{q} \kappa_{i}^{\rho_{i}}\left(1-\kappa_{i}\right)^{s \gamma_{i}}\right]\left[\prod_{1 \leqslant i<j \leqslant q}\left(\kappa_{i}-\kappa_{j}\right)^{\gamma_{i} \gamma_{j}}\right],
$$

where the $\rho_{i}$ are integers. Since $\kappa_{1}, \ldots, \kappa_{q}$ are distinct with $0<\left|\kappa_{i}\right|<1$, we see that $\operatorname{det} E \neq 0$; thus the only solution to (2.22) is the trivial one, namely, $\sigma_{i, k}=0$. Consequently, $g_{\nu}$ of (2.21) vanishes, which means that we. have failed to construct a nontrivial eigensolution of $\left(G-z_{0} I\right) g=0$ that belongs to $H$. Hence, $z_{0}$ is not an eigenvalue of $G$, and Kreiss' Main Theorem assures stability.

We have proven the following result.

THEOREM (KREISS, 1965). Let the initial value problem

$$
u_{t}=a u_{x} ; \quad a=\text { const; } \quad x(\operatorname{sig} a) \geqslant 0, t \geqslant 0 ; \quad u(x, 0)=f(x),
$$

be approximated by a dissipative, strongly-stable, two-sided scheme, which is complemented by outflow extrapolation of arbitrary accuracy, at the boundary $x=0$. Then, the overall approximation is stable.

We conclude this section by demonstrating the cases $r=1$ and $r=2$, which cover all schemes of practical importance. In particular, $r=1, r=2$, agree, respectively, with two schemes which we employ in Section 3.

For $r=1$, we have to extrapolate only at one point, $\mu=0$, and the characteristic equation has only one root $\kappa$ inside the unit disc. So, an eigensolution of $(G-z) g=0,|z| \geqslant 0, z \neq 1$, must be of the form

$$
g_{\nu}=\sigma \kappa^{\nu}, \quad \nu \geqslant 0
$$

and substituting this solution into the boundary condition (2.19), we obtain the single equation

$$
\left[\sum_{j=0}^{s}\left(\begin{array}{l}
s \\
j
\end{array}\right)(-1)^{j^{j}}\right]^{j} \sigma=0
$$

Here

$$
\operatorname{det} E=E=\sum_{j=0}^{s}\left(\begin{array}{l}
s \\
j
\end{array}\right)(-1)^{j^{j}} \kappa^{j}=(1-\kappa)^{s} \neq 0
$$

and stability follows. 
When $r=2$, we use at each time step, $v_{1}(t), \ldots, v_{s}(t)$, to compute $v_{0}(t)$; and then $v_{0}(t), \ldots, v_{s-1}(t)$, to determine $v_{-1}(t)$. Equation (2.17) has now two roots $\kappa_{i}$ with $0<\left|\kappa_{i}\right|<1$, and we distinguish between two possibilities. The first is $\kappa_{1}=$ $\kappa_{2}=\kappa$, where

$$
g_{\nu}=\sigma_{1} \kappa^{\nu}+\sigma_{2} \nu \kappa^{\nu}, \quad \nu \geqslant-1 .
$$

The insertion of (2.27) into (2.19) yields the system of two equations

$$
\sum_{j=0}^{s}\left(\begin{array}{l}
s \\
j
\end{array}\right)(-1)^{j}\left[\sigma_{1}+(\mu+j) \sigma_{2}\right] \kappa^{\mu+j}=0, \quad \mu=0,-1
$$

Writing (2.28) in the form of $E \sigma=0$, with $\sigma^{\prime}=\left(\sigma_{1}, \sigma_{2}\right)$ being the transposed unknown vector, we find that

$$
\operatorname{det} E=-\kappa^{-1}(1-\kappa)^{2 s} \neq 0 .
$$

The second possibility is $\kappa_{1} \neq \kappa_{2}$. Here

$$
g_{\nu}=\sigma_{1} \kappa_{1}^{\nu}+\sigma_{2} \kappa_{2}^{\nu}, \quad \nu \geqslant-1,
$$

which we substitute into (2.19) to obtain

$$
\sum_{j=0}^{s}\left(\begin{array}{l}
s \\
j
\end{array}\right)(-1)^{j}\left(\sigma_{1} \kappa_{1}^{j+\mu}+\sigma_{2} \kappa_{2}^{j+\mu}\right)=0, \quad \mu=0,-1 .
$$

The coefficient determinant is

$$
\operatorname{det} E=\kappa_{1}^{-1} \kappa_{2}^{-1}\left(1-\kappa_{1}\right)^{s}\left(1-\kappa_{2}\right)^{s}\left(\kappa_{1}-\kappa_{2}\right) \neq 0,
$$

and again, by the Main Theorem, stability follows.

3. Numerical Results. In this section we consider the initial value test-problem

$$
u_{t}=u_{x} ; \quad x \geqslant 0, t \geqslant 0 ; u(x, 0)=\sin 2 \pi x,
$$

whose analytic solution is

$$
u(x, t)=\sin 2 \pi(x+t) .
$$

We begin by writing down the second order accurate Lax-Wendroff scheme (L-W), [7], which for the linear equation in (3.1), takes the form

$$
\begin{gathered}
v_{\nu}^{m+1}=\sum_{j=-1}^{1} a_{j} v_{\nu+j}^{m}, \quad v_{\nu}^{m} \equiv v_{\nu}(m \Delta t), \\
a_{0}=1-\lambda^{2}, \quad a_{ \pm 1}=1 / 2\left(\lambda^{2} \pm \lambda\right), \quad \lambda=\Delta t / \Delta x .
\end{gathered}
$$

We recall (e.g. [8, Chapter 12]), that in the above case $(a=1)$,

$$
\lambda<1
$$

is a condition which assures dissipativity and strong stability.

Here, $r=1$; hence, to approximate (3.1), we need to specify only one boundary value, $v_{0}^{m}$. Extrapolating via $v_{1}^{m}, \ldots, v_{s}^{m}$, we get, according to (2.11), a boundary condition

$$
v_{0}^{m}=\sum_{j=1}^{s}\left(\begin{array}{l}
s \\
j
\end{array}\right)(-1)^{j+1} v_{j}^{m}
$$


which is of (local) accuracy of order $s$. By the theorem of Section 2, the algorithm defined in (3.3) together with (3.5), remains stable, provided (3.4) is satisfied.

For the numerical computations we set an artificial boundary at $x=b, b>0$, where we use values of the analytic solution. Nevertheless, we restrict attention to results in the interval $0 \leqslant x \leqslant 1$, so we choose a large enough $b$, in order to secure that during the integration period, $0 \leqslant t \leqslant 1$, there will be no interaction between the boundary at $x=b$, and the numerical solution at $0 \leqslant x \leqslant 1$. In other words, errors due to the right boundary, which propagate inward, never reach the region $0 \leqslant x \leqslant 1$.

\begin{tabular}{lccccc}
\hline \multicolumn{1}{c}{$\Delta x$} & $s$ & $m$ & $\|e\|_{(0,1)}$ & $m$ & \multicolumn{1}{c}{$t=1}$. \\
\hline .05 & 2 & 20 & $2.75-2$ & 40 & $5.57-2$ \\
.025 & 2 & 40 & $6.93-3$ & 80 & $1.39-2$ \\
.0125 & 2 & 80 & $1.72-3$ & 160 & $3.46-3$ \\
.05 & 1 & 20 & $5.63-2$ & 40 & $7.03-2$ \\
.025 & 1 & 40 & $1.98-2$ & 80 & $2.23-2$ \\
.0125 & 1 & 80 & $7.00-3$ & 160 & $7.44-3$ \\
\hline
\end{tabular}

TABLE 1. L-W results with $\lambda=\Delta t / \Delta x=1 / 2$. $m=t / \Delta t$ is the number of time steps; $\alpha-n$ presents $\alpha \cdot 10^{-n}$.

The quantity $\|e\|_{(0,1)}$ in Table 1 is the $H$-norm of the error, restricted to $0 \leqslant x$ $\leqslant 1$, i.e., in our case $(r=1)$,

$$
\|e\|_{(0,1)}^{2}=\|e(t)\|_{(0,1)}^{2}=\sum_{\nu=0}^{J}\left[v_{\nu}(t)-u\left(x_{\nu}, t\right)\right]^{2} \Delta x, \quad J=\frac{1}{\Delta x} .
$$

The integer $s$ indicates the order of extrapolation: $s=1$ and $s=2$ mean constant and linear extrapolation, respectively. We realize that all the results are stable. As expected, linear extrapolation maintains the overall second order accuracy of the L-W scheme, while constant extrapolation reduces the total accuracy.

Our second check relates to a centered 5-point scheme suggested by Gottlieb and Turkel (G-T), [4]. We consider the family of schemes in (2.4) of [2], set its parameters to be $\alpha=1 / 2, \sigma=1$, and linearize. The approximation we get for Eq. (3.1) is

$$
\begin{gathered}
v_{\nu}^{m+1}=\sum_{j=-2}^{2} a_{j} v_{\nu+j}^{m}, \quad a_{0}=1-\frac{7}{4} \lambda^{2}, \\
a_{ \pm 1}=\lambda\left(\lambda \pm \frac{2}{3}\right), a_{ \pm 2}=-\frac{\lambda}{4}\left(\frac{\lambda}{2} \pm \frac{1}{3}\right), \quad \lambda=\frac{\Delta t}{\Delta x} .
\end{gathered}
$$

It was shown in [2], that (3.8) is stable if and only if

$$
\lambda \leqslant \sqrt{2} / 2
$$


and if we somewhat sharpen this condition and require

$$
\lambda<\sqrt{2} / 2 \text {, }
$$

we have dissipativity as well.

Unlike the L-W case, which is of second order accuracy both in time and space, the G-T approximation is of second order accuracy in time and fourth order accuracy in space. By this we mean that the truncation error $\epsilon$ satisfies

$$
\epsilon=\Delta t\left[O\left(\Delta t^{2}\right)+O\left(\Delta t \cdot \Delta x^{2}\right)+O\left(\Delta x^{4}\right)\right] \leqslant \Delta t\left[O\left(\Delta t^{2}\right)+O\left(\Delta x^{4}\right)\right] .
$$

Such schemes-see also the Kreiss-Oliger approximation, [5]-have advantages when dealing with problems whose solutions have strong space variations but vary slowly in time. In particular, this concept fits problems which approach a steady state. These ideas were discussed in [6].

In the G-T case $r=2$, so we need to specify $v_{0}(t), v_{-1}(t)$. Again, by (2.11) we have

$$
v_{\mu}^{m}=\sum_{j=1}^{s}\left(\begin{array}{l}
s \\
j
\end{array}\right)(-1)^{j+1} v_{j+\mu}^{m}, \quad \mu=0,-1,
$$

\begin{tabular}{|c|c|c|c|c|c|c|}
\hline \multirow[b]{2}{*}{$\Delta x$} & \multirow[b]{2}{*}{$\lambda$} & \multirow[b]{2}{*}{$s$} & \multicolumn{2}{|c|}{$t=.5$} & \multicolumn{2}{|c|}{$t=1$} \\
\hline & & & $m$ & $\|e\|_{(0,1)}$ & $m$ & $\|e\|_{(0,1)}$ \\
\hline .05 & .5 & 4 & 20 & $9.42-3$ & 40 & $1.85-2$ \\
\hline .025 & .25 & 4 & 80 & $5.75-4$ & 160 & $1.16-3$ \\
\hline .05 & .5 & 3 & 20 & $2.14-2$ & 40 & $2.42-2$ \\
\hline .025 & .25 & 3 & 80 & $1.78-3$ & 160 & $1.92-3$ \\
\hline
\end{tabular}

where it is understood that the evaluation of $v_{0}^{m}$ precedes $v_{-1}^{m}$.

TABLE 2. G-T results.

Table 2 shows that the results are stable. Here, in analogy with the previous case,

$$
\|e\|_{(0,1)}^{2} \equiv \sum_{\nu=-1}^{J}\left[v_{\nu}(t)-u\left(x_{\nu}, t\right)\right] \Delta x, \quad J=\frac{1}{\Delta x} .
$$

Since G-T is of fourth order accuracy in space, it is expected that utilizing a cubic extrapolation $(s=4)$, will maintain the 4-space accuracy. As shown, results for $\Delta x$ $=.05$ and $\Delta t=.025$ are compared with those for $\Delta x / 2$ and $\Delta t / 4$. Indeed, the error is reduced by a factor of 16 . This ratio is destroyed if we use quadratic extrapolation.

As a final point of reference to the figures in both tables, we mention that the norm of the numerical solution was for all times $0 \leqslant t \leqslant 1$,

$$
\|v\|_{(0,1)}^{2} \equiv \sum_{\nu=-r}^{J} v_{\nu}(t)^{2} \Delta x \sim 0.5, \quad J=\frac{1}{\Delta x} .
$$


Department of Mathematics

University of California

Los Angeles, California 90024

1. M. GOLDBERG \& S. ABARBANEL, "Stable approximations for hyperbolic systems with moving internal boundary conditions," Math. Comp., v. 28, 1974, pp. 413-447; corrigenda, ibid., v. 29, 1975, p. 1167 . MR 52 \#2240.

2. D. GOTTLIEB \& E. TURKEL, Dissipative Two-Four Methods for Time Dependent Problems, ICASE Report 75-22, NASA Langley Research Center, Hampton, Virginia, 1975.

3. B. GUSTAFFSON, "The convergence rate for difference approximations to mixed initial boundary value problems," Math. Comp., v. 29, 1975, pp. 396-406. MR 52 \#7154.

4. H.-O. KREISS, "Difference approximations for hyperbolic differential equations," $\mathrm{Nu}$ merical Solution of Partial Differential Equations, (Proc. Sympos., Univ. Maryland, 1965), Academic Press, New York, 1966, pp. 51-58. MR 34 \#7039.

5. H.-O. KREISS, "Stability theory for difference approximations of mixed initial boundary value problems. I," Math. Comp., v. 22, 1968, pp. 703-714. MR 39 \#2355.

6. H.-O. KREISS \& J. OLIGER, "Comparison of accurate methods for the integration of hyperbolic equations," Tellus, v. 24, 1972, pp. 199-215. MR 47 \#7926.

7. P. D. LAX \& B. WENDROFF, "Systems of conservation laws," Comm. Pure Appl. Math., v. 13, 1960, pp. 217-237. MR 22 \#11523.

8. R. D. RICHTMYER \& K. W. MORTON, Difference Methods for Initial-Value Problems, 2nd ed., Interscience Tracts in Pure and Appl. Math., no. 4, Wiley, New York, 1967. MR 36 \#3515. 